Article

\title{
Antimicrobial Susceptibility Pattern of Porcine Respiratory Bacteria in Spain
}

\author{
Anna Vilaró ${ }^{1}$, Elena Novell ${ }^{1}$, Vicens Enrique-Tarancón ${ }^{1}$, Jordi Balielles ${ }^{1}$, Carles Vilalta ${ }^{2}$, \\ Sonia Martinez ${ }^{3}$ and Lorenzo José Fraile Sauce ${ }^{4, *(D)}$ \\ 1 Grup de Sanejament Porcí, 25192 Lleida, Spain; micro@gsplleida.net (A.V.); elena@gsplleida.net (E.N.); \\ Vicens@gsplleida.net (V.E.-T); Jordi@gsplleida.net (J.B.) \\ 2 Freelance researcher, Arbeca, 25192 Lleida, Spain; cvilalta@umn.edu \\ 3 Departamento de Sanidad Animal, Universidad de León, 24006 León, Spain; smarm@unileon.es \\ 4 Departament de Ciència Animal, Escola Tècnica Superior d'Enginyeria Agrària, University of \\ Lleida-Agrotecnio, 25198 Lleida, Spain \\ * Correspondence: lorenzo.fraile@ca.udl.cat; Tel.: +34-973702814
}

Received: 19 June 2020; Accepted: 9 July 2020; Published: 11 July 2020

check for updates

\begin{abstract}
The monitoring of antimicrobial susceptibility of pig pathogens is critical to optimize antimicrobial treatments and prevent development of resistance with a one-health approach. The aim of this study was to investigate the antimicrobial susceptibility patterns of swine respiratory pathogens in Spain from 2017 to 2019. Bacterial isolation and identification were carried out following standardized methods from samples coming from sacrificed or recently deceased pigs with acute clinical signs compatible with respiratory tract infections. Minimum inhibitory concentration (MIC) values were determined using the broth microdilution method containing a total of 10 and 7-8 antimicrobials/concentrations respectively, in accordance with the recommendations presented by the Clinical and Laboratory Standards Institute (CLSI). The obtained antimicrobial susceptibility varies between pig respiratory pathogens. Actinobacillus pleuropneumoniae (APP) and Pasteurella multocida (PM) were highly susceptible ( $\geq 90 \%$ ) to ceftiofur, florfenicol and macrolides (tilmicosin, tildipirosin and tulathromycin). However, the antimicrobial susceptibility was intermediate ( $>60 \%$ but $<90 \%$ ) for amoxicillin and enrofloxacin in the case of APP and sulfamethoxazole/trimethropim and tiamulin in the case of PM. Both bacteria showed low $(<60 \%)$ antimicrobial susceptibility to doxycycline. Finally, Bordetella bronchiseptica was highly susceptible only to tildipirosin and tulathromycin $(100 \%)$ and its susceptibility for florfenicol was close to $50 \%$ and $<30 \%$ for the rest of the antimicrobial families tested. These results emphasize the need of determining antimicrobial susceptibility in pig respiratory cases in order to optimize the antimicrobial treatment in a case-by-case scenario.
\end{abstract}

Keywords: antimicrobial susceptibility; swine; respiratory pathogens

\section{Introduction}

The Porcine Respiratory Disease Complex (PRDC) is a syndrome that results from a combination of infectious and non-infectious factors [1]. Actinobacillus pleuropneumoniae (APP), Pasteurella multocida (PM), Mycoplasma hyopneumoniae (MH), Bordetella bronchiseptica (BB) and Glaesserella (Haemophilus) parasuis (GP) are the most common bacterial agents involved. Porcine reproductive and respiratory syndrome virus (PRRSV), swine influenza virus (SIV) and porcine circovirus type 2 virus (PCV2) are the most prevalent viral agents [1-6]. On the other hand, many non-infectious predisposing factors are also involved in PRDC, such as poor environmental conditions, density, stressors, season of the year, genetic background and production flow (all-in-all out versus continuous flow) [7-9]. As a general approach, preventive medicine programs should be based on applying measures to 
control PRDC in a cost-effective way, such as improving environmental conditions, decreasing density and stressors, combined with vaccination against the major viral and bacterial infectious etiologic factors [10]. However, if such measures are not in place or fail, the use of antimicrobials may be needed.

The use of antimicrobials could be necessary to control bacteria involved in PRDC with a therapeutic or metaphylactic (group medication) goal. In particular, the objective of antimicrobial therapy is to provide an effective drug to obtain a fast, clinical recovery from the infection in affected animals but reducing the probability of generating antimicrobial resistance [11]. However, its use is one of the factors involved in the emergence and spread of bacterial antimicrobial resistance (AR) from pig origin worldwide [12,13]. Resistant bacteria in humans, food, environment and animals are interconnected, and exchange may continuously take place between these ecological niches. For this reason, AR needs to be addressed with a one-health perspective and action plans have been adopted to address this problem [14]. These plans are based on the development of programs to monitor the usage of antimicrobial agents in pig medicine and the occurrence of antimicrobial resistance in pigs at the European level $[15,16]$. In veterinary medicine, Antimicrobial Susceptibility Testing (AST) data could predict the clinical outcome of antimicrobial treatment, allowing a rational choice of these drugs to treat bacterial infections [11,17]. Antimicrobial susceptibility is usually measured using minimum inhibitory concentration (MIC) that is the lowest antimicrobial concentration that inhibits the growth of the target bacteria in vitro. Moreover, it is necessary to have valid clinical breakpoints (CB) to correctly interpret the MIC value obtained for each clinical case. Thus, all the clinical cases with MIC values below CB could be correctly treated with one antimicrobial at the common registered dose [11].

The Spanish national program to control antimicrobial resistance has been adopted to reduce the risk of developing antimicrobial resistance since 2014 [16]. One of the points of the program is focused on reducing the antimicrobial consumption in livestock in order to reduce the prevalence of resistant bacteria. This antimicrobial consumption has been steadily decreasing in the last years according to the available European data [15]. Unfortunately, there is little knowledge of antimicrobial susceptibility patterns for animal pathogenic bacteria in Spain. In this study, we present antimicrobial susceptibility patterns for some of the most important pig respiratory pathogenic bacteria, collected during the period 2017-2019 in Spain.

\section{Results}

Four-hundred samples were received from sow, wean-to-finish and fattening farms across Spain suffering clinical respiratory cases. In the case of sow farms, the samples were obtained from their nursery facilities. Bacterial isolation (APP, PM and BB) was only possible in 80.3\% (321/400) of the cases, and in 22\% (88/400) of them, it was possible to isolate more than one bacteria. The isolation of APP and PM and PM and BB was possible in 17.5\% (70/400) and 4.5\% (18/400) of the cases, respectively.

$\mathrm{MIC}_{50}, \mathrm{MIC}_{90}$ and antimicrobial susceptibility for 162, 130 and 29 strains of APP, PM and BB are described in Tables 1-3, respectively. The number of GP isolates was low (35 strains) and it was not possible to determine the MIC value because we were unable to grow GP strains with the microdilution technique in our laboratory. The MIC distribution observed for each microorganism and drug are shown in Figures 1-5. 
Table 1. Bordetella bronchiseptica (BB), $\mathrm{MIC}_{50}, \mathrm{MIC}_{90}$, current recommended clinical breakpoints (CB) and antimicrobial susceptibility. The $\mathrm{MIC}_{50}$ and $\mathrm{MIC}_{90}$ were determined from the MIC distribution from 29 BB strains isolated from respiratory clinical cases. The antimicrobial susceptibility was calculated as the percentage of bacterial isolates below $\mathrm{CB}$.

\begin{tabular}{ccccc}
\hline Antimicrobial & $\mathbf{M I C}_{\mathbf{5 0}}(\boldsymbol{\mu g} / \mathbf{m L})$ & $\mathbf{M I C}_{\mathbf{9 0}}(\boldsymbol{\mu g} / \mathbf{m L})$ & $\begin{array}{c}\text { Clinical } \\
\text { Breakpoint (CB) } \\
(\boldsymbol{\mu g} / \mathbf{m L})\end{array}$ & $\begin{array}{c}\text { Antimicrobial } \\
\text { Susceptibility } \\
\text { Based On CB }\end{array}$ \\
\hline Amoxicillin & 16 & 16 & 0.5 & 0 \\
Ceftiofur & 4 & 4 & 2 & 0 \\
Doxycycline & 1 & 2 & 0.5 & 27.7 \\
Enrofloxacin & 0.5 & 0.5 & 0.25 & 20.7 \\
Florfenicol & 2 & 4 & 2 & 51.7 \\
Sulfamethoxazole/ & 4 & 8 & 0.5 & 3.4 \\
trimethropim \& & 64 & 64 & 16 & 0 \\
Tiamulin & 4 & 8 & 8 & 100 \\
Tildipirosin & 32 & 64 & 16 & 27.6 \\
Tilmicosin & 8 & 8 & 16 & 100 \\
Tulathromycin & & & & 0.4 \\
\hline
\end{tabular}

${ }^{1}$ Florfenicol, tildipirosin and tulathromycin CB were obtained from CLSI M100 2018 and CLSI VETO8 4th ed., 2018. The rest of the CB were extrapolated from Pasteurella multocida (Table 3). \& MIC represented in the table is for trimethropin. Sulfamethoxazole/trimethropim ratio tested is 19:1.

A

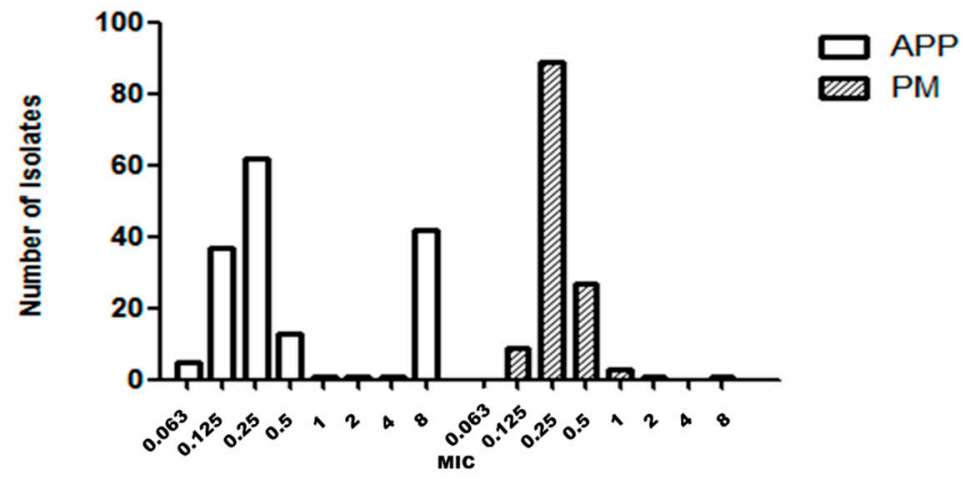

B

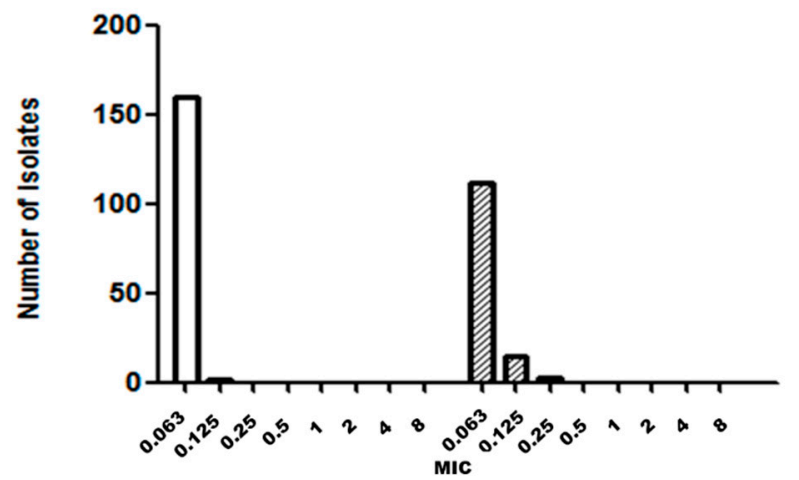

Figure 1. Minimum inhibitory concentration (MIC, $\mu \mathrm{g} / \mathrm{mL}$ ) distribution of amoxicillin (A) and ceftiofur (B) for Actinobacillus pleuropneumoniae (APP) and Pasteurella multocida (PM) isolated from lungs of pigs with respiratory symptoms. 
A

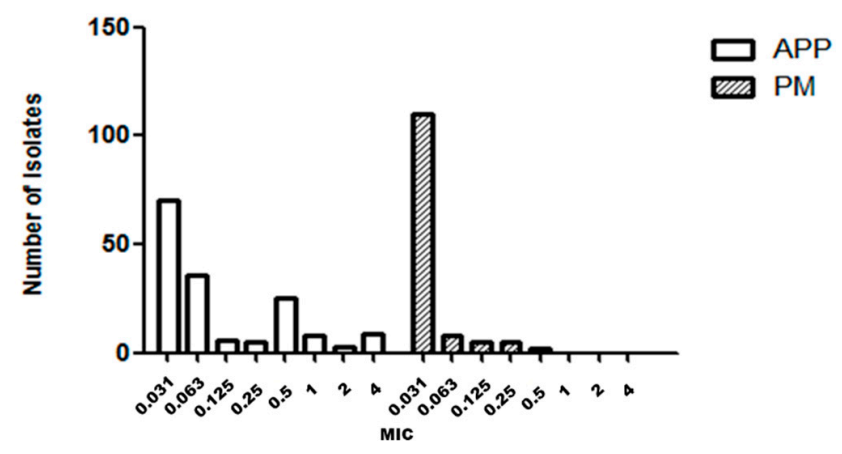

B

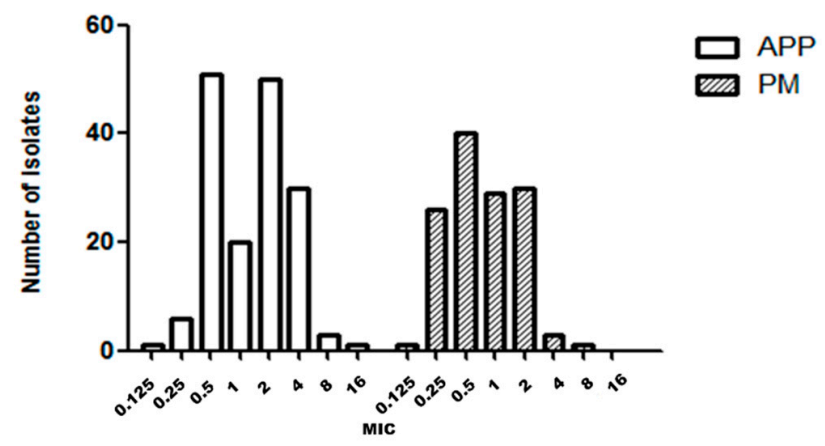

Figure 2. Minimum inhibitory concentration (MIC, $\mu \mathrm{g} / \mathrm{mL}$ ) distribution of enrofloxacin (A) and doxycycline (B) for Actinobacillus pleuropneumoniae (APP) and Pasteurella multocida (PM) isolated from lungs of pigs with respiratory symptoms.

A
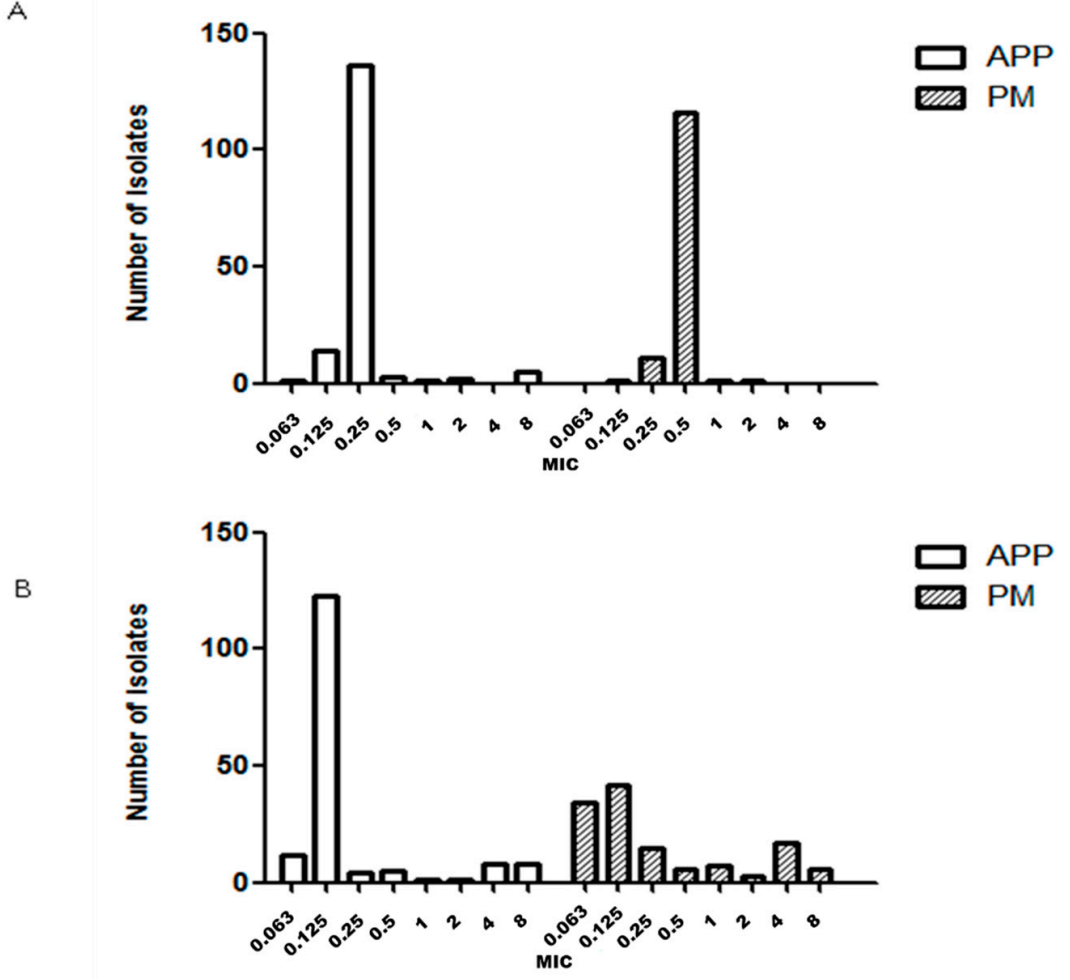

Figure 3. Minimum inhibitory concentration (MIC, $\mu \mathrm{g} / \mathrm{mL}$ ) distribution of florfenicol (A) and sulfamethoxazole/trimethoprim (B) for Actinobacillus pleuropneumoniae (APP) and Pasteurella multocida (PM) isolated from lungs of pigs with respiratory symptoms. In the case of sulfametoxazole/trimethoprim, the MIC value for trimethoprim is represented. 
A

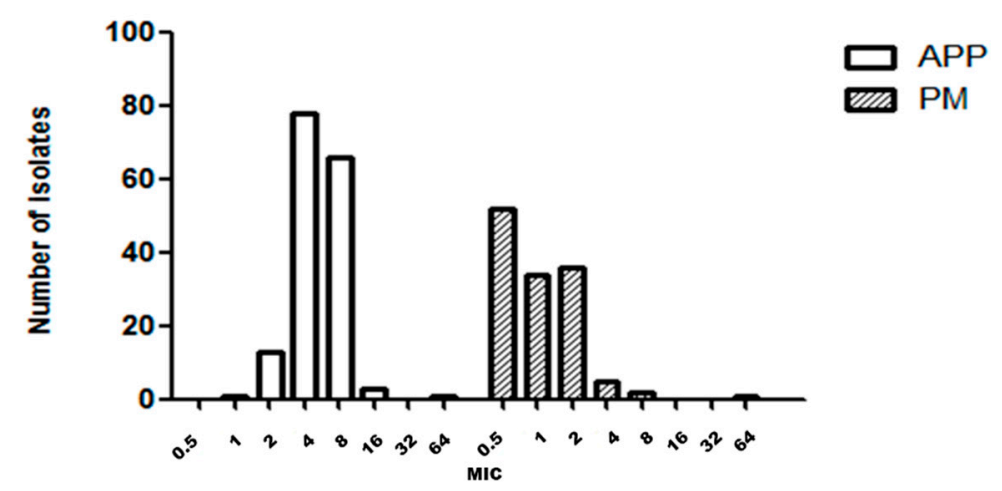

B

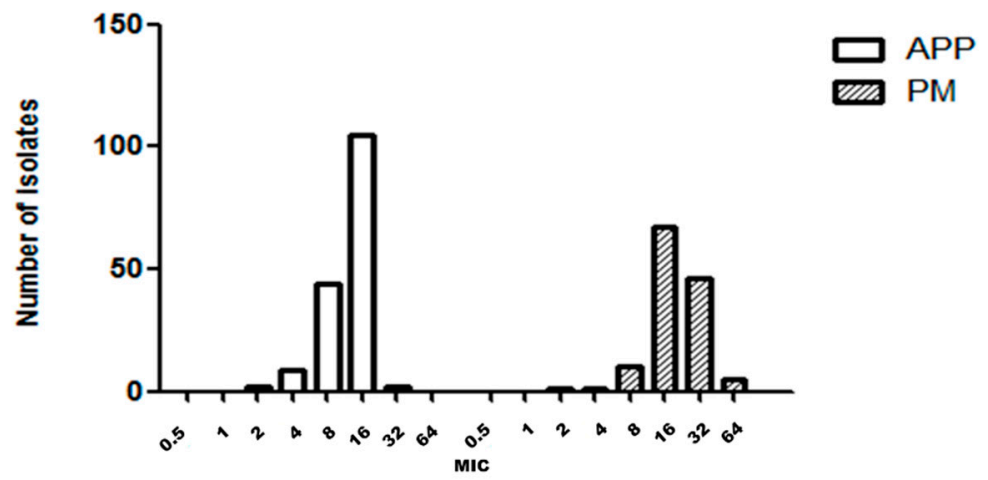

Figure 4. Minimum inhibitory concentration (MIC, $\mu \mathrm{g} / \mathrm{mL}$ ) distribution of tildipirosin (A) and tiamulin (B) for Actinobacillus pleuropneumoniae (APP) and Pasteurella multocida (PM) isolated from lungs of pigs with respiratory symptoms.

A

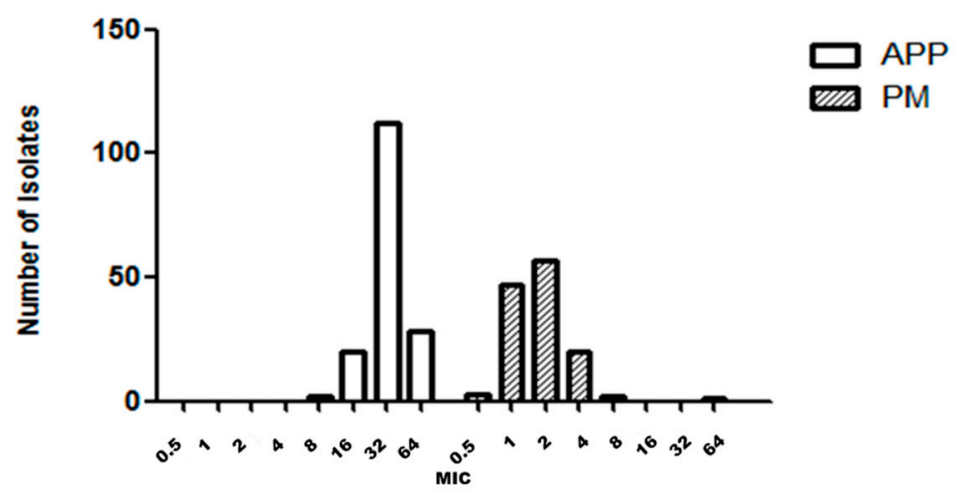

B

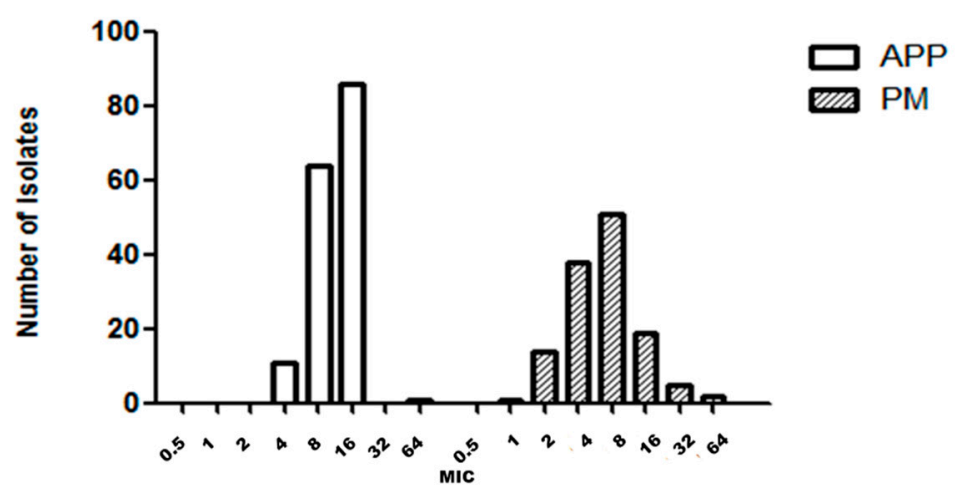

Figure 5. Minimum inhibitory concentration (MIC, $\mu \mathrm{g} / \mathrm{mL}$ ) distribution of tulathromycin (A) and tilmicosin (B) for Actinobacillus pleuropneumoniae (APP) and Pasteurella multocida (PM) isolated from lungs of pigs with respiratory symptoms. 
APP and PM MIC distributions were very similar for ceftiofur (Figure 1B) and florfenicol (Figure 3A). On the other hand, the APP and PM MIC distributions were different for the following antimicrobials: amoxicillin (Figure 1A), enrofloxacin (Figure 2A), sulfamethoxazole/trimethoprim (Figure 3B), doxycycline (Figure 2B), tildipirosin and tiamulin (Figure 4A,B) and tulathromycin and tilmicosin (Figure 5A,B).

The isolates of APP were highly susceptible $(\geq 90 \%)$ to macrolides (tildipirosin, tulathromycin and tilmicosin), tiamulin, florfenicol, sulfamethoxazole/trimethropim and ceftiofur. However, the antimicrobial susceptibility was intermediate (around $72 \%$ ) for amoxicillin and enrofloxacin and low (35.7\%) for doxycycline. Pasteurella multocida showed high susceptibility ( $\geq 90 \%)$ to macrolides (tildipirosin, tulathromycin and tilmicosin), florfenicol, enrofloxacin, amoxicillin and ceftiofur. However, PM antimicrobial susceptibility was intermediate $(74.7 \%)$ for sulfamethoxazole/trimethropim and tiamulin (60.8\%) and low (51.5\%) for doxycycline. Thus, in general terms, APP and PM were susceptible to many families of antimicrobials, whereas BB was highly susceptible (100\%) only to tildipirosin and tulathromycin. On the other hand, BB susceptibility for florfenicol was close to $50 \%$ and $<30 \%$ for the rest of the antimicrobial families tested (Tables 1-3).

\section{Discussion}

There is a scarcity of updated information about antimicrobial susceptibility among porcine pathogens in Spain because there is no official program for their surveillance. The current study aims to address this gap by determining antimicrobial susceptibility, through determining MICs, of ten antimicrobials to three major respiratory tract pathogens recovered, prior to antibiotic treatment, from diseased pigs across Spain. In this case, we have focused on the antimicrobials most frequently used to treat respiratory disease in pigs.

The measurement of antimicrobial susceptibility is carried out by MIC determination that is more reproducible and comparable between laboratories [11] than disk diffusion techniques due to concerns about disk quality, performance issues $[18,19]$ and variability intrinsically associated to some antimicrobials for the disk diffusion technique [20]. The MIC is the lowest antimicrobial concentration that inhibits in vitro the growth of the target bacteria in specific conditions of in vitro incubation. In this study, the antimicrobial susceptibility has been determined using international guidelines on antimicrobial susceptibility determination [21,22] that cannot be directly compared with other studies only based on disk diffusion techniques [20]. This methodology does not emulate the natural biophase in which bacteria grow in vivo, such as blood, interstitial or intracellular fluid. In any case, antimicrobial sensitivity testing in vitro is used to provide information concerning the efficacy of antimicrobial agents in vivo and thus determine whether an antibiotic is suitable or not to treat a specific condition [23], but MIC determination, as any technique, has weaknesses that should be outweighed [24]. Thus, there is an interesting scientific discussion about the usefulness of MIC to foresee the clinical outcome. Some authors recently have proposed to consider both pathogen- (MIC) and patient-specific drug exposure information to predict treatment success in humans. If both pieces of information are taken into account, it will change how antimicrobials are selected and it will allow optimizing the treatment through precision medicine [25]. Unfortunately, this approach is far away from the "everyday" veterinary medicine, where posology regimens of antimicrobials are fixed for each animal species and bacteria to be treated (registered dose for a veterinary medicinal product). Furthermore, the selection of the antimicrobial is even stricter due to the existence of withdrawal periods in livestock to assure food safety that exclude off-label use of antimicrobials to apply precision medicine [25].

Comparison of antimicrobial susceptibility from other laboratories must be carried out with caution due to inconsistencies in methodology (MIC versus disk diffusion technique), selection of antimicrobial substances in the test panel and variations in interpretation criteria for clinical breakpoints. In our study, the isolates of APP were highly susceptible for macrolides (tildipirosin, tilmicosin and tulathromycin), tiamulin, florfenicol, sulfamethoxazole/trimethropim and ceftiofur. 
However, the antimicrobial susceptibility was intermediate for amoxicillin and enrofloxacin, and low for doxycycline. This antimicrobial susceptibility pattern described for APP in this study agrees with results obtained by Spanish researchers with strains collected from 1997 [26]. These results could be surprising due to the historic consumption of antimicrobials in Spain and the authors recommend not directly linking the use of antimicrobials with the presence of antimicrobial resistance for any drug-microorganism combination. Moreover, our results are quite similar for isolates from other European countries with some differences $[27,28]$. Overall, there are still good opportunities to treat infections by APP with antimicrobials, but the presence of strains resistant to doxycycline, amoxicillin and enrofloxacin in Spain highlight the importance of monitoring antimicrobial susceptibility and select the most suitable antimicrobial in a case-by-case situation.

In our study, the isolates of PM were highly susceptible for macrolides (tildipirosin, tilmicosin and tulathromycin), florfenicol, enrofloxacin, amoxicillin and ceftiofur. However, the antimicrobial susceptibility was intermediate for sulfamethoxazole/trimethropim and tiamulin, and low for doxycycline. Thus, the antimicrobial susceptibility pattern is different from that described for APP in spite of the fact that both bacteria are respiratory ones. Again, these results highlight that monitoring of antimicrobial susceptibility must be carried out for each drug-microorganism combination. Moreover, the antimicrobial susceptibility pattern described in our study is very similar to the pattern described by Spanish researchers with strains collected from 1987 [29] for macrolides (>97\%), ceftiofur (100\%), ampicillin (98\%), enrofloxacin (100\%), tiamulin (50\%) and florfenicol $(100 \%)$, and by European researchers in a multi-country study to determine the antimicrobial susceptibility of PM in pigs for ceftiofur (100\%), enrofloxacin (100\%), florfenicol $(99.3 \%)$, tetracycline $(65.8 \%)$ and macrolides ( $>90 \%$ ) [30]. Thus, the antimicrobial susceptibility of PM seems to have not changed significantly across time, at least, in Europe. This result could be surprising taking into account the enormous variability in the consumption of antimicrobials in livestock across Europe and the authors recommend, again, not directly linking the use of antimicrobials with the presence of antimicrobial resistance for any drug-microorganism combination.

Bordetella bronchiseptica causes a mild or non-progressive inflammation in the nasal cavity that usually needs no treatment. However, if the bacterium is co-infecting with toxigenic PM, it can lead to severe progressive atrophic rhinitis [31]. Moreover, BB may cause pneumonia in young piglets in some cases. In our study, the number of BB strains was quite low to precisely define their MIC distributions. Thus, our results of antimicrobial susceptibility for BB must be interpreted with caution. Moreover, there is a lack of approved clinical breakpoints for many antimicrobials with this bacterium, making comparison with other studies extremely complicated. Bordetella bronchiseptica has been described to be intrinsically resistant to ampicillin due to production of beta-lactamases [32,33] and our results agree with this affirmation not only for amoxicillin but also for ceftiofur. In our case, the isolates had extremely high MIC values for doxycycline that exclude them as a therapeutic option to treat BB infection. This lack of antimicrobial susceptibility agrees with Speakman et al. [34] who described a plasmid-encoded tetracycline resistance gene, tet $C$, for this bacterium. Finally, macrolides are also the most susceptible family against BB in Denmark [28], and Dayao et al. [35] also reported no resistance to tulathromycin in Australia. 
Table 2. Actinobacillus pleuropneumoniae (APP), $\mathrm{MIC}_{50}, \mathrm{MIC}_{90}$, current recommended clinical breakpoints (CB) and antimicrobial susceptibility. The $\mathrm{MIC}_{50}$ and $\mathrm{MIC}_{90}$ were determined from the MIC distribution from 162 APP strains isolated from respiratory clinical cases. The antimicrobial susceptibility was calculated as the percentage of bacterial isolates below CB.

\begin{tabular}{ccccc}
\hline Antimicrobial & $\mathbf{M I C}_{\mathbf{5 0}}(\boldsymbol{\mu g} / \mathbf{m L})$ & $\mathbf{M I C}_{\mathbf{9 0}}(\boldsymbol{\mu g} / \mathbf{m L})$ & $\begin{array}{c}\text { Clinical } \\
\text { Breakpoint (CB) } \\
(\boldsymbol{\mu g} / \mathbf{m L})\end{array}$ & $\begin{array}{c}\text { Antimicrobial } \\
\text { Susceptibility } \\
\text { Based on CB }\end{array}$ \\
\hline Amoxicillin & 0.25 & 16 & $0.5^{\$}$ & 72.2 \\
Ceftiofur & 0.06 & 0.06 & 2 & 100 \\
Doxycycline & 1 & 4 & $0.5^{+}$ & 35.7 \\
Enrofloxacin & 0.06 & 1 & 0.25 & 72.2 \\
Florfenicol & 0.25 & 0.25 & 2 & 97.0 \\
Sulfamethoxazole/ & 0.125 & 2 & 0.5 & 88.9 \\
trimethropim \& & 16 & 16 & 16 & 98.8 \\
Tiamulin & 4 & 8 & 16 & 99.4 \\
Tildipirosin & 8 & 16 & 16 & 99.4 \\
Tilmicosin & 32 & 64 & 64 & 100 \\
Tulathromycin & & & & \\
\hline
\end{tabular}

${ }^{1}$ All clinical breakpoints were obtained from Clinical and Laboratory Standards Institute (CLSI) M100 2018 and CLSI VETO8 4th ed., 2018, with the following clarifications: ${ }^{\$}$ Schwarz et al. (2008) [36]. ${ }^{+}$Extrapolated from tetracycline. \& MIC represented in the table is for trimethropin. Sulfamethoxazole/trimethropim ratio tested is 19:1.

Table 3. Pasteurella multocida (PM), $\mathrm{MIC}_{50}, \mathrm{MIC}_{90}$, current recommended clinical breakpoints (CB) and antimicrobial susceptibility. The $\mathrm{MIC}_{50}$ and $\mathrm{MIC}_{90}$ were determined from the MIC distribution from 130 PM strains isolated from respiratory clinical cases. The antimicrobial susceptibility was calculated as the percentage of bacterial isolates below $\mathrm{CB}$.

\begin{tabular}{ccccc}
\hline Antimicrobial & $\mathbf{M I C}_{\mathbf{5 0}}(\boldsymbol{\mu g} / \mathbf{m L})$ & $\mathbf{M I C}_{\mathbf{9 0}}(\mu \mathrm{g} / \mathbf{m L})$ & $\begin{array}{c}\text { Clinical } \\
\text { Breakpoint (CB) } \\
(\mu \mathrm{g} / \mathbf{m L})\end{array}$ & $\begin{array}{c}\text { Antimicrobial } \\
\text { Susceptibility } \\
\text { Based On CB }\end{array}$ \\
\hline Amoxicillin & 0.25 & 0.5 & $0.5^{\$}$ & 96.2 \\
Ceftiofur & 0.06 & 0.12 & 2 & 100 \\
Doxycycline & 0.5 & 4 & 0.5 & 51.5 \\
Enrofloxacin & 0.03 & 0.06 & 0.25 & 98.5 \\
Florfenicol & 0.5 & 0.5 & 2 & 100 \\
Sulfamethoxazole/ & 0.12 & 4 & 0.5 & 74.7 \\
trimethropim \& & 16 & 32 & 16 & 60.8 \\
Tiamulin & 1 & 2 & 4 & 97.7 \\
Tildipirosin & 8 & 16 & 16 & 94.6 \\
Tilmicosin & 2 & 4 & 16 & 100 \\
Tulathromycin & & 4 & & \\
\hline
\end{tabular}

${ }^{1}$ All clinical breakpoints were obtained from CLSI M100 2018 and CLSI VETO8 4th ed., 2018 with the following clarifications: $\$$ Schwarz et al. (2008) [36]. ${ }^{+}$Extrapolated from tetracycline. \& MIC represented in the table is for trimethropin. Sulfamethoxazole/trimethropim ratio tested is 19:1.

It is necessary to have valid information about $\mathrm{CB}$ to correctly interpret the MIC value obtained in each clinical case. Thus, all the clinical cases with MIC values below $\mathrm{CB}$ could be treated with the antimicrobial, at the registered dose, with a high success rate. Unfortunately, there are no clinical veterinary breakpoints available for all the antimicrobials and bacteria for pigs. In our study, we have used well-established CLSI clinical breakpoints for seven out of ten antimicrobials. However, CLSI veterinary breakpoints for sulfamethoxazole/trimethropim and Pasteurellaceae have not been set. In this study, the CLSI CB available for Streptococcus suis $(0.5 \mu \mathrm{g} / \mathrm{mL})$ have been used. This value exactly agrees with the CB used by El Garch et al. [30] in a study to monitor the antimicrobial susceptibility for sulfamethoxazole/trimethropim of porcine pathogens in Europe, making results directly comparable. These authors carried out this extrapolation due to the high similarity between Haemophilus influenziae and respiratory pathogens in pigs. Moreover, The CLSI clinical breakpoint for amoxicillin $(0.5 \mu \mathrm{g} / \mathrm{mL})$ 
has been obtained from the literature [36]. This CB value for amoxicillin is equal to the CLSI CB for ampicillin that belongs to the same antimicrobial family (beta-lactam antimicrobials). However, Rey et al. [37] proposed that the CB breakpoint (obtained through pharmacokinetics/pharmacodynamic analysis) for amoxicillin, administered by the intramuscular route, could be as low as $0.125 \mu \mathrm{g} / \mathrm{mL}$ for pig respiratory pathogens. If we had used this proposed breakpoint instead of the chosen one $(0.5 \mu \mathrm{g} / \mathrm{mL})$, the percentage of antimicrobial susceptibility for APP and PM would have been $26 \%$ and $7 \%$ respectively, which is extremely different from the results shown for this antimicrobial in this paper. Thus, there is an urgent need to have CLSI CB breakpoints available for every antimicrobial/bacteria and feedback from swine practitioners when using these antimicrobials at the registered dose [38]. In this sense, the collaboration between microbiologists, pharmacologists and swine practitioners is highly recommended. Finally, CB for doxycycline was extrapolated from CLSI CB available for tetracycline and porcine respiratory pathogens. In general, the percentage of antimicrobial susceptibility determined in our study is comparable with any other study published using CLSI clinical breakpoints. In the case of doxycycline, amoxicillin and sulfamethoxazole/trimethropim, our results must be compared checking the CB used by other authors before making direct comparisons between them. Finally, antimicrobial susceptibility pattern can change with time [28] and this is one of the main reasons to determine it across time in order to select the most suitable antimicrobial, taking into account efficacy criteria and the one-health approach [14].

\section{Materials and Methods}

\subsection{Clinical Samples}

Samples were drawn from diseased or recently deceased pigs from farms across Spain showing acute clinical signs of respiratory tract infections that had not been exposed to antimicrobial treatment for, at least, 15 days prior to sampling between the years 2017 and 2019. Thus, the sampled animals were between 3 and 24 weeks old, the pigs had overt clinical respiratory signs with or without depression and/or hyperthermia $\left(>39.8^{\circ} \mathrm{C}\right)$ and the mortality rate increased significantly, versus the previous baseline situation, due mainly to respiratory causes at farm level. For each clinical case, samples of lungs of two recently deceased pigs $(<12 \mathrm{~h}$ ) were submitted under refrigeration to the laboratory (to increase possibility to isolation). If, during the veterinary visit, there were no recently dead pigs suitable for sampling, at least two animals with acute respiratory signs were humanely sacrificed and lung samples were drawn. In any case, only one isolate per animal/herd was included in the study. All experimental procedures were approved by the Ethics Committee for Animal Experimentation of the University of Lleida and performed in accordance with authorization 10343 issued by the Catalan Department of Agriculture, Livestock, Fisheries and Food (Section of biodiversity and hunting).

\subsection{Bacterial Isolation and Identification}

Clinical specimens were cultured aseptically onto blood agar (Columbia agar with 5\% Sheep blood, 254005 BD), chocolate agar (GC II agar with IsoVitaleX, 254060, BD, Franklin Lakes NJ, USA)) and MacConkey agar (4016702, Biolife Italiana Srl, Milano, Italy) and incubated at $35-37^{\circ} \mathrm{C}$ in aerobic conditions with $5-10 \% \mathrm{CO}_{2}$ for $24-48$ hours. Identification of isolates (APP, PM, BB and GP) was carried out by matrix-assisted laser desorption ionization-time of flight (MALDI-TOF Biotyper System, Bruker Daltonics, Bremen, Germany). Individual strains were stored at $-80{ }^{\circ} \mathrm{C}$ in brain heart infusion (CM1135, Oxoid, Madrid, Spain) with 30\% of glycerol (G9012, Sigma-Aldrich, Madrid, Spain).

\subsection{Antimicrobial Sensitivity Testing}

MIC values were determined using the broth microdilution method by means of customized 96-well microtiter plates (Sensititre, Trek diagnostic Systems Inc., East Grinstead, UK) containing a total of 10 and 7-8 antimicrobials/concentrations respectively, in accordance with the recommendations presented by the Clinical and Laboratory Standards Institute (CLSI) [21,22]. The antimicrobials tested 
included amoxicillin, ceftiofur, doxycycline, enrofloxacin, florfenicol, sulfamethoxazole/trimethoprim, tiamulin, tilmicosin, tildipirosin and tulathromycin. This antimicrobial panel was selected to represent common compounds licensed for treatment of pig respiratory diseases in practice.

Bacteria were thawed, cultured on chocolate agar and incubated at $35-37^{\circ} \mathrm{C}$ in ambient air (or with $5-10 \% \mathrm{CO}_{2}$ for APP) for 18-24 h. Three to five colonies were picked and emulsified in demineralized water (or cation-adjusted Mueller-Hinton broth (CAMHB) for APP) to obtain a turbidity of $0.5 \mathrm{McF}$ arland standard (Sensititre ${ }^{\mathrm{TM}}$ nephelometer V3011). Suspensions were further diluted in CAMHB (for PM and BB) or Veterinary Fastidious Medium (in the case of APP) to reach a final inoculum concentration of $5 \times 10^{5}$ colony forming units ( $\mathrm{cfu}$ )/mL (Table 4). Then, the Sensititre panel was reconstituted by adding $100 \mu \mathrm{L} /$ well of the inoculum. Plates containing PM and BB isolates were incubated at $35 \pm 2{ }^{\circ} \mathrm{C}$ for $18-20 \mathrm{~h}$. In the case of APP isolates, plates were covered with a perforated seal and incubated at $35 \pm 2{ }^{\circ} \mathrm{C}$, with $5-10 \% \mathrm{CO}_{2}$ for $20-24 \mathrm{~h}$ [21,22]. The antibiotic panels were read manually using Sensititre ${ }^{\mathrm{TM}}$ Vizion (V2021) and the MIC value was established as the lowest drug concentration inhibiting visible growth. For each strain tested, a colony count and a purity check were performed following CLSI and manufacturer recommendations. Moreover, quality control strains were also included in all susceptibility testing runs. Thus, Actinobacillus pleuropneumoniae (ATCC $27090^{\mathrm{TM}}$ ) and Escherichia coli (ATCC $25922^{\mathrm{TM}}$ ) were included as quality control [21,22]. The MICs of the quality control strains had to be within acceptable CLSI ranges to accept the results obtained in the laboratory.

Table 4. Details of the conditions used to carry out minimum inhibitory concentration (MIC) determination using the broth microdilution method by means of customized 96-well microtiter plates (Sensititre, Trek diagnostic Systems Inc., East Grinstead, UK).

\begin{tabular}{|c|c|c|c|c|c|}
\hline Microorganism & $\begin{array}{l}0.5 \text { McFarland } \\
\text { Suspension } \\
\text { Medium }\end{array}$ & Broth & $\begin{array}{c}\text { Final } \\
\text { Inoculum }\end{array}$ & $\begin{array}{c}\text { Plate } \\
\text { Reconstitution }\end{array}$ & $\begin{array}{l}\text { Incubation } \\
\text { Conditions }\end{array}$ \\
\hline $\begin{array}{c}\text { Pasteurella } \\
\text { multocida and } \\
\text { Bordetella } \\
\text { bronchiseptica }\end{array}$ & Water & CAMHB & $5 \times 10^{5} \mathrm{cfu} / \mathrm{mL}$ & $100 \mu \mathrm{L}$ & $\begin{array}{c}35 \pm 2{ }^{\circ} \mathrm{C} \\
18-24 \mathrm{~h} \\
\text { Non-CO } \\
\text { incubator }\end{array}$ \\
\hline $\begin{array}{l}\text { Actinobacillus } \\
\text { pleuropneumoniae }\end{array}$ & САMHB & VFM & $5 \times 10^{5} \mathrm{cfu} / \mathrm{mL}$ & $100 \mu \mathrm{L}$ & $\begin{array}{c}35 \pm 2{ }^{\circ} \mathrm{C} \\
20-24 \mathrm{~h} \\
\mathrm{CO}_{2} \text { incubator } \\
\text { perforated seal }\end{array}$ \\
\hline
\end{tabular}

CAMHB—Cation-adjusted Mueller-Hinton Broth. VFM—Veterinary Fastious Medium. cfu—colony forming units.

\subsection{Data Analysis}

All the clinical cases with MIC values below CB were classified as susceptible because they could be treated with the antimicrobial, at the registered dose, with a high success rate. The results of the sensitivity tests are presented as MIC distributions and these were determined for each species-antimicrobial combination. $\mathrm{MIC}_{50}$ and $\mathrm{MIC}_{90}$ were defined as MICs inhibiting $50 \%$ and $90 \%$ of the strains, respectively. Clinical breakpoints from CLSI were used [21,22] to determine antimicrobial susceptibility. However, CLSI veterinary breakpoints for sulfamethoxazole/trimethropim and Pasteurellaceae have not been set. Thus, the CLSI CB available for Streptococcus suis $(0.5 \mu \mathrm{g} / \mathrm{mL})$ and sulfamethoxazole/trimethropim have been used in this study. The clinical breakpoint for amoxicillin $(0.5 \mu \mathrm{g} / \mathrm{mL})$ has been obtained from the literature [36] and CLSI CB available for tetracycline $(0.5 \mu \mathrm{g} / \mathrm{mL})$ and porcine respiratory pathogens was extrapolated for doxycycline [21,22]. The antimicrobial susceptibility was considered high at levels $\geq 90 \%$ and low at levels $\leq 60 \%$, as described by Holmer et al. [28]. 


\section{Conclusions}

The obtained antimicrobial susceptibility varies between pig respiratory pathogens. Actinobacillus pleuropneumoniae and Pasteurella multocida were highly susceptible to ceftiofur, florfenicol and macrolides. However, the antimicrobial susceptibility was intermediate for amoxicillin and enrofloxacin in the case of APP and sulfamethoxazole/trimethropim and tiamulin in the case of PM. Both bacteria showed low antimicrobial susceptibility to doxycycline. Finally, Bordetella bronchiseptica was highly susceptible only to tildipirosin and tulathromycin and its susceptibility for florfenicol was close to $50 \%$ and $<30 \%$ for the rest of the antimicrobial families tested. These results emphasize the need for determining antimicrobial susceptibility in pig respiratory cases in order to optimize the antimicrobial treatment in a case-by-case scenario and provide a robust criteria to select the most suitable antimicrobials, taking into account the one-health approach. On the other hand, there is an urgent need to have CLSI CB breakpoints available for every antimicrobial/bacteria and feedback from swine practitioners when using these antimicrobials at the registered dose.

Author Contributions: Conceptualization, A.V. and L.J.F.S.; methodology, A.V., E.N., V.E.-T., J.B., S.M. and C.V.; formal analysis, L.J.F.S and C.V.; writing - original draft preparation, L.J.F.S. and A.V.; writing-review and editing, L.J.F.S., C.V. and A.V.; funding acquisition, E.N. and V.E.-T. All authors have read and agreed to the published version of the manuscript.

Funding: This research received no external funding.

Acknowledgments: This study was carried out with the support of the Porcine Sanitation Group of Lleida, Spain (Grup de Sanejament Porci-GSP), in collaboration with veterinary clinicians working in the field. One part of this research was supported by Cost Action CA18217: European Network for Optimization of Veterinary Antimicrobial Treatment. We are very thankful to Dr César B. Gutiérrez Martín for revising the manuscript.

Conflicts of Interest: The authors declare no conflict of interest.

\section{References}

1. Brockmeier, S.L.; Halbur, P.G.; Thacker, E.L. Porcine respiratory disease complex. In Polymicrobial Diseases; Brogden, K.A., Guthmiller, J.M., Eds.; ASM Press: Washington, DC, USA, 2002.

2. Fraile, L.; Alegre, A.; López-Jiménez, R.; Nofrarías, M.; Segalés, J. Risk factors associated with pleuritis and cranio-ventral pulmonary consolidation in slaughter-aged pigs. Vet. J. 2010, 18, 326-333. [CrossRef] [PubMed]

3. Fablet, C.; Marois-Crehan, C.; Simon, G.; Grasland, B.; Jestin, A.; Kobisch, M.; Madec, F.; Rose, N. Infectious agents associated with respiratory diseases in 125 farrow-to-finish pig herds: A cross sectional study. Vet. Microb. 2012, 157, 152-163. [CrossRef] [PubMed]

4. Van Alstine, W.G. Respiratory system. In Diseases of Swine; Zimmerman, J.J., Karriker, L.A., Kent, A.R., Schwartz, J., Stevenson, G.W., Eds.; Wiley-Blackwell: Ames, IA, USA, 2012.

5. Maes, D.; Sibila, M.; Kuhnert, P.; Segalés, J.; Haesebrouck, F.; Pieters, M. Update on Mycoplasma hyopneumoniae infections in pigs: Knowledge gaps for improved disease control. Transbound. Emerg. Dis. 2018, 65, 110-124. [CrossRef] [PubMed]

6. Sassu, E.L.; Bossé, J.T.; Tobias, T.J.; Gottschalk, M.; Langford, P.R.; Hennig-Pauka, I. Update on Actinobacillus pleuropneumoniae-Knowledge, gaps and challenges. Transbound. Emerg. Dis. 2018, 65, 72-90. [CrossRef] [PubMed]

7. Opriessnig, T.; Giménez-Lirola, L.G.; Halbur, P.G. Polymicrobial respiratory disease in pigs. Anim. Health Res. Rev. 2011, 12, 133-148. [CrossRef]

8. Colomer, M.À.; Margalida, A.; Fraile, L. Improving the management procedures in farms infected with the Porcine Reproductive and Respiratory Syndrome virus using PDP models. Sci. Rep. 2019, 9, 1-13. [CrossRef]

9. Khatun, A.; Nazki, S.; Jeong, C.G.; Gu, S.; Mattoo, S.U.S.; Lee, S.I.; Yang, M.S.; Lim, B.; Kim, K.S.; Kim, B.; et al. Effect of polymorphisms in porcine guanylate-binding proteins on host resistance to PRRSV infection in experimentally challenged pigs. Vet. Res. 2020, 51,1-14. [CrossRef]

10. Sargeant, J.M.; Deb, B.; Bergevin, M.D.; Churchill, K.; Dawkins, K.; Dunn, J.; Hu, D.; Moody, C.; O'Connor, A.M.; O'Sullivan, T.L.; et al. Efficacy of bacterial vaccines to prevent respiratory disease in swine: A systematic review and network meta-analysis. Anim. Health Res. Rev. 2019, 20, 274-290. [CrossRef] 
11. Fraile, L. Antimicrobial Therapy in Swine. Practical Approach; Editorial Servet: Zaragoza, Spain, 2013.

12. Bronzwaer, S.L.A.M.; Cars, O.; Buchholz, U.; Mölstad, S.; Goettsch, W.; Veldhuijzen, I.K.; Kool, J.I.; Sprenger, M.J.W.; Degener, J.E. The relationship between antimicrobial use and antimicrobial resistance in Europe. Emerg. Infect. Dis. 2002, 8, 278-282. [CrossRef]

13. Aarestrup, F.M.; Duran, C.O.; Burch, D.G.S. Antimicrobial resistance in swine production. Anim. Health Res. Rev. 2008, 9, 135-148. [CrossRef]

14. World Health Organization. Global Action Plan on Antimicrobial Resistance. Available online: https://www.who.int/antimicrobial-resistance/global-action-plan/en/ (accessed on 1 June 2020).

15. European Surveillance of Veterinary Antimicrobial Consumption (ESVAC). Sales of Veterinary Antimicrobial Agents in 29 European Countries in 2017, Trends from 2011 to 2017. Ninth ESVAC Report. 2019. Available online: https://www.ema.europa.eu/en/documents/report/sales-veterinary-antimicrobial-agents31-european-countries-2017_en.pdf (accessed on 1 June 2020).

16. Agencia Española De Medicamentos Y Productos Sanitarios. Plan Estratégico y de Acción Para Reducir el Riesgo de Selección y Diseminación de la Resistencia a los Antibióticos, 2019. Plan Nacional Resistencia a Antibióticos (PRAN). Available online: https://www.aemps.gob.es/laAEMPS/planificacion-AEMPS/docs/ Plan-estrategico-2019-2022.pdf. (accessed on 1 June 2020).

17. European Commission. Guidelines for the Prudent Use of Antimicrobials in Veterinary Medicine (2015/C299/04). 2015. Available online: https:/ec.europa.eu/health/sites/health/files/antimicrobial_resistance/ docs/2015_prudent_use_guidelines_en.pdf. (accessed on 1 June 2020).

18. Humphries, R.M.; Kircher, S.; Ferrell, A.; Krause, K.M.; Malherbe, R.; Hsiung, A.; Burnham, C.A. The Continued Value of Disk Diffusion for Assessing Antimicrobial Susceptibility in Clinical Laboratories: Report from the Clinical and Laboratory Standards Institute Methods Development and Standardization Working Group. J. Clin. Microbiol. 2018, 56, 1-10. [CrossRef] [PubMed]

19. Humphries, R.M.; Hindler, J.A.; Shaffer, K.; Campeau, S.A. Evaluation of Ciprofloxacin and Levofloxacin Disk Diffusion and Etest Using the 2019 Enterobacteriaceae CLSI Breakpoints. J. Clin. Microbiol. 2019, 57, 1-7. [CrossRef] [PubMed]

20. Aguirre, L.; Vidal, A.; Seminati, C.; Tello, M.; Redondo, N.; Darwich, L.; Martín, M. Antimicrobial resistance profile and prevalence of extended-spectrum beta-lactamases (ESBL), AmpC beta-lactamases and colistin resistance ( $\mathrm{mcr}$ ) genes in Escherichia coli from swine between 1999 and 2018. Porcine Health Manag. J. 2020, 6, 1-6. [CrossRef] [PubMed]

21. Clinical and Laboratory Standards Institute. CLSI Supplement VET08. Performance Standards for Antimicrobial Disk and Dilution Susceptibility Tests for Bacteria Isolated from Animals, 4th ed.; Clinical and Laboratory Standards Institute: Wayne, PA, USA, 2018.

22. Clinical and Laboratory Standards Institute. CLSI Supplement M100. Performance Standards for Antimicrobial Susceptibility Testing, 28th ed.; Clinical and Laboratory Standards Institute: Wayne, PA, USA, 2018.

23. Wen, X.; Gehring, R.; Stallbaumer, A.; Riviere, J.E.; Volkova, V.V. Limitations of MIC as sole metric of pharmacodynamic response across the range of antimicrobial susceptibilities within a single bacterial species. Sci. Rep. 2016, 6, 1-8. [CrossRef]

24. Mouton, J.W.; Muller, A.E.; Canton, R.; Giske, C.G.; Kahlmeter, G.; Turnidge, J. MIC-based dose adjustment: Facts and fables. J. Antimicrob. Chemother. 2018, 73, 564-568. [CrossRef]

25. Bader, J.C.; Lakota, E.A.; Andes, D.R.; Rubino, C.M.; Ambrose, P.G.; Bhavnani, S.M. Time for Precision: A World Without Susceptibility Breakpoints. Open Forum Infect. Dis. 2018, 5, 1-6. [CrossRef]

26. Gutiérrez-Martín, C.B.; del Blanco, N.; Blanco, M.; Navas, J.; Rodríguez-Ferri, E.F. Changes in antimicrobial susceptibility of Actinobacillus pleuropneumoniae isolated from pigs in Spain during the last decade. Vet. Microbiol. 2006, 115, 218-222. [CrossRef]

27. Hendriksen, R.S.; Mevius, D.J.; Schroeter, A.; Teale, C.; Jouy, E.; Butaye, P.; Franco, A.; Utinane, A.; Amado, A.; Moreno, M.; et al. Occurrence of antimicrobial resistance among bacterial pathogens and indicator bacteria in pigs in different European countries from year 2002-2004: The ARBAO-II study. Acta Vet. Scand. 2008, 50, 1-10. [CrossRef]

28. Holmer, I.; Salomonsen, C.M.; Jorsal, S.E.; Astrup, L.B.; Jensen, V.F.; Høg, B.B.; Pedersen, K. Antibiotic resistance in porcine pathogenic bacteria and relation to antibiotic usage. BMC Vet. Res. 2019, 15, 1-13. [CrossRef] 
29. Lizarazo, Y.A.; Ferri, E.F.; de la Fuente, A.J.; Martín, C.B. Evaluation of changes in antimicrobial susceptibility patterns of Pasteurella multocida subsp multocida isolates from pigs in Spain in 1987-1988 and 2003-2004. Am. J. Vet. Res. 2006, 67, 663-668. [CrossRef]

30. El Garch, F.; de Jong, A.; Simjee, S.; Moyaert, H.; Klein, U.; Ludwig, C.; Marion, H.; Haag-Diergarten, S.; Richard-Mazet, A.; Thomas, V.; et al. Monitoring of antimicrobial susceptibility of respiratory tract pathogens isolated from diseased cattle and pigs across Europe, 2009-2012: VetPath results. Vet. Microbiol. 2016, 194, 1-22. [CrossRef]

31. Zhao, Z.; Wang, C.; Xue, Y.; Tang, X.; Wu, B.; Cheng, X.; He, Q.; Chen, H. The occurrence of Bordetella bronchiseptica in pigs with clinical respiratory disease. Vet. J. 2011, 188, 337-340. [CrossRef] [PubMed]

32. Kadlec, K.; Kehrenberg, C.; Wallmann, J.; Schwarz, S. Antimicrobial susceptibility of Bordetella bronchiseptica isolates from porcine respiratory tract infections. Antimicrob. Agents Chemother. 2004, 48, 4903-4906. [CrossRef]

33. Prüller, S.; Rensch, U.; Meemken, D.; Kaspar, H.; Kopp, P.A.; Klein, G.; Kehrenberg, C. Antimicrobial susceptibility of Bordetella bronchiseptica isolates from swine and companion animals and detection of resistance genes. PLoS ONE 2015, 10, 1-14. [CrossRef]

34. Speakman, A.J.; Binns, S.H.; Osborn, A.M.; Corkill, J.E.; Kariuki, S.; Saunders, J.R.; Dawson, S.; Gaskell, R.M.; Hart, C.A. Characterization of antibiotic resistance plasmids from Bordetella bronchiseptica. J. Antimicrob. Chemother. 1997, 40, 811-816. [CrossRef] [PubMed]

35. Dayao, D.A.; Gibson, J.S.; Blackall, P.J.; Turni, C. Antimicrobial resistance in bacteria associated with porcine respiratory disease in Australia. Vet. Microbiol. 2014, 171, 232-235. [CrossRef] [PubMed]

36. Schwarz, S.; Böttner, A.; Goossens, L.; Hafez, H.M.; Hartmann, K.; Kaske, M.; Kehrenberg, C.; Kietzmann, M.; Klarmann, D.; Klein, G.; et al. A proposal of clinical breakpoints for amoxicillin applicable to porcine respiratory tract pathogens. Vet. Microbiol. 2008, 126, 178-188. [CrossRef] [PubMed]

37. Rey, J.F.; Laffont, C.M.; Croubels, S.; De Backer, P.; Zemirline, C.; Bousquet, E.; Guyonnet, J.; Ferran, A.A.; Bousquet-Melou, A.; Toutain, P.L. Use of Monte Carlo simulation to determine pharmacodynamic cutoffs of amoxicillin to establish a breakpoint for antimicrobial susceptibility testing in pigs. Am. J. Vet. Res. 2014, 75, 124-131. [CrossRef]

38. Burch, D.G.S.; Sperling, D. Amoxicillin-current use in swine medicine. J. Vet. Pharmacol. Ther. 2018, 41, 356-368. [CrossRef] 